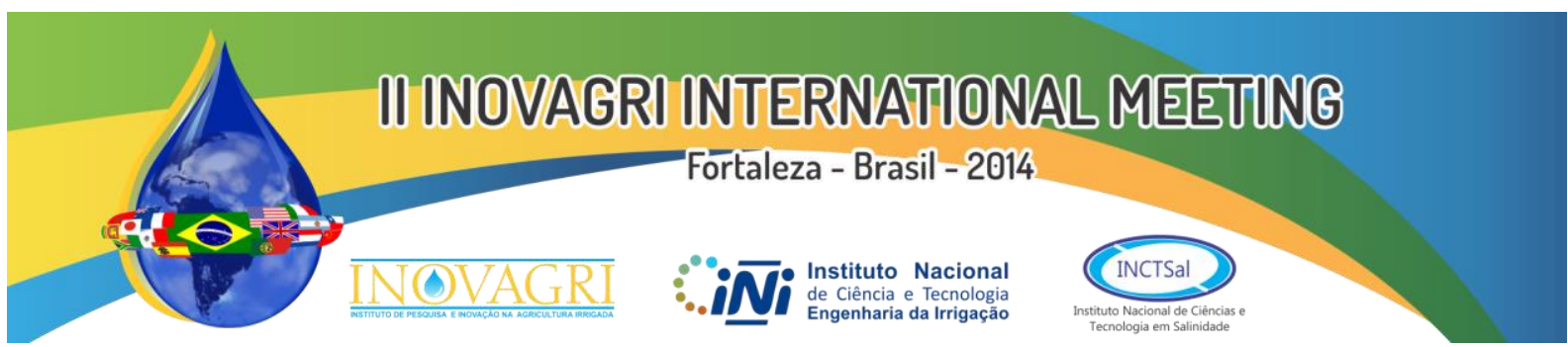

http://dx.doi.org/10.12702/ii.inovagri.2014-a684

\title{
ÁGUA DISPONÍVEL NO SOLO COM PONTO DE MURCHA PERMANENTE OBTIDO POR DIFERENTES MÉTODOS
}

\author{
T. da S. Felisberto ${ }^{1}$, L. B. Marinho ${ }^{2}$; M. D. Deon ${ }^{3}$, E. de A. Andrade ${ }^{1}$; J. R. de Souza Filho ${ }^{1}$
}

RESUMO: O objetivo do trabalho foi comparar os valores de água disponível no solo com o ponto de murcha permanente (PMP), determinado pelo método da centrífuga ou pelo método fisiológico, com a capacidade de campo (CC) estimada por diferentes potenciais mátricos $(\Psi \mathrm{m})$. Pelo método da centrífuga foram determinadas a umidade na $\mathrm{CC}$ nos potenciais mátricos de -0,006 e -0,01 MPa e no PMP de -1,5 MPa. O PMP fisiológico foi obtido utilizando a Célosia cultivada em ambiente protegido. Estas foram irrigadas diariamente a fim de manter o solo com umidade próxima à capacidade de vaso até a floração, quando foi suspensa a irrigação. Ao apresentar sintomas de murcha, as mudas foram levadas ao laboratório e colocadas em local escuro e saturado, o procedimento foi repetido até a murcha irreversível, quando determinou-se a umidade residual do solo pelo método gravimétrico. Houve efeito significativo do método, determinação do PMP e do $\Psi \mathrm{m}$ adotado na obtenção da CC para quantificação da água disponível no solo a $1 \%$ de probabilidade, contudo não se verificou efeito significativo da interação entre os fatores. O maior valor de água disponível foi ao considerar a planta como indicadora de murcha permanente, sendo que a Celósia extraiu água a potencial mátrico inferior a -1,5 MPa. A água disponível no solo aumentou ao utilizar o maior $\Psi m$ na determinação da CC.

PALAVRAS-CHAVE: retenção de água, floricultura, déficit hídrico.

\section{AVAILABLE WATER CAPACITY IN SOIL WITH PERMANENT WILTING POINT MEASURED BY DIFFERENT METHODS}

SUMMARY: The aim of this study was to compare the measures of available water capacity comparing the permanent wilting point ( PWP) determined by the centrifuge or by the physiological method, and field capacity ( FC ) estimated by different matric potentials ( $\Psi \mathrm{m}$ ). By the centrifuge method it was determined soil moisture at FC in matric potentials of -0.006 and $-0.01 \mathrm{MPa}$ and at the PWP at $-1.5 \mathrm{MPa}$. The physiological PWP was obtained using Celosia cultivated in a greenhouse. They were watered daily to maintain the soil moisture at pot capacity until blooming, when the irrigation was halted. When plants presented symptoms of wilting, they were taken to the laboratory and placed in a dark, saturated site. The procedure was repeated until occurrence of irreversible wilting when we determined the residual soil moisture by the gravimetric method. There was a significant effect of method, matric potential adopted in determining the PWP and $\Psi \mathrm{m}$ adopted in obtaining the FC for quantification of available water capacity of soil at $1 \%$ probability, however there was no significant interaction between factors. The largest amount of water available occurred when the plant was used as an indicator of PWP, and the celosia extracted water less than $-1.5 \mathrm{MPa}$ matric potential. The available water capacity estimation of soil increased when using the larger $\Psi \mathrm{m}$ in determining the FC.

KEYWORDS : water retention, floriculture, water deficit .

\footnotetext{
${ }^{1}$ Discente, Engenharia agronômica, Depto Tecnologia e Ciências Sociais, av. Edgard Chastinet, São Geraldo, CEP $48905-$ 680, Juazeiro, BA. Fone (74) 3611-7363. E-mail: thomazvilbe@ hotmail.com

${ }^{2}$ Profa., Doutora, Depto Tecnologia e Ciências Sociais, UNEB, Juazeiro, BA.

${ }^{3}$ Doutor, Pesquisador Embrapa Cpatsa, Petrolina, PE.
} 


\section{INTRODUÇÃO}

A técnica de irrigação depende de uma boa relação entre a estimativa da disponibilidade da água no solo e a habilidade das plantas em utilizar a água armazenada no solo.

A água disponível no solo para as culturas é considerada como o intervalo entre os valores de umidade na capacidade de campo (CC) e ponto de murcha permanente (PMP). Em laboratório a umidade na CC pode ser determinada utilizando-se funis de Büchner, a mesa de tensão e o sistema de câmara de pressão de Richards. Na literatura encontram-se indicações de que a CC é a água retida a um potencial mátrico ( $\Psi \mathrm{m})$ de -0,033 MPa para solos argilosos (Colman, 1947) e de -0,01MPa para solos de textura grosseira (Cassel \& Nielsen, 1986). Contudo, para solos arenoso segundo Rivers \& Shipp (1978) a CC ocorre em um potencial de água ainda maior, ou seja, igual a -0,0067 MPa.

O PMP é atingido quando a umidade do solo proporciona a murcha irreversível das plantas e não há mais a recuperação da turgescência das folhas quando as plantas são colocadas num ambiente escuro e saturado de umidade (Briggs \& Shantz, 1912).

Existem diferentes métodos, indiretos (câmara de Richards, psicrômetro WP4, Centrífuga, entre outros) e diretos (fisiológico), que definem o PMP. Dentre eles, o mais preciso é também o mais laborioso e demorado - a determinação do PMP fisiológico ou direto, aplicada por Briggs \& Shantz (1912). Segundo estes autores o ponto de murcha permanente representa a umidade mínima disponível às plantas no solo. Richards \& Weaver (1943) determinaram a umidade no PMP pelo método do laboratório e pelo método fisiológico e observaram que o $\Psi \mathrm{m}$ de $-1,5 \mathrm{MPa}$ correspondeu $\pm 1,5 \%$ de teor de água gravimétrica, obtido pelo método fisiológico, em mais de 100 solos testados. A partir destes resultados, indicaram que a determinação em laboratório da umidade ao $\Psi \mathrm{m}$ de -1,5 MPa é uma maneira prática de se determinar o limite inferior de disponibilidade de água às plantas.

Contudo, as plantas podem atingir o PMP a potencial mátrico do solo dentro da ampla faixa entre -0,8 e -3 MPa, conforme citado por Klein et al., (2006). Estes autores, ao comparar a determinação da disponibilidade de água no solo e ponto de murcha fisiológico obtiveram teores de água menores para o PMP pelo método fisiológico que pela Câmara de Richards, ao utilizar as culturas do milho e girassol como plantas teste. Já em um estudo usando melão como planta indicadora em Neossolo Quartzarênico, Costa et. al., (2009) encontraram maiores umidade do solo no PMP significativamente determinado pelo método fisiológico comparado que pela retenção de água no $\Psi m$ de -1,50 MPa, o que indica ser a cultura do melão sensível ao estresse hídrico.

Trabalho realizado por Salvestro (2013) indica que não existe uma medida em laboratório que possa ser, no campo, um real substituto do PMP uma vez que a capacidade de retenção de água dos solos varia principalmente de acordo com a classe textural, e salienta que determinadas plantas podem absorver água sob potenciais mátricos do solo inferiores a -1,5 MPa.

De acordo com o exposto, verifica-se a demanda de informações sobre a relação entre o PMP obtido pelos métodos diretos e indiretos sob diferentes condições de cultivo, pois erros em sua determinação podem acarretar perdas de produção pela inadequada quantificação da demanda hídrica e/ ou manejo da irrigação. Apesar da importância deste tema poucos trabalhos têm sido realizados no Brasil e, principalmente em regiões semiáridas.

Por isso, o objetivo do trabalho foi obter e comparar os valores de água disponível no solo com PMP determinado pelo método da centrífuga e fisiológico e a CC estimada por dois diferentes $\Psi \mathrm{m}$.

\section{MATERIAL E MÉTODOS}

Para obtenção e comparação da água disponível no solo com ponto de murcha permanente determinado pelo método da centrífuga e fisiológico utilizando a espécie ornamental Celósia (Celosia argentea $\mathrm{L}$.) como indicadora.

No Laboratório de Solos da Embrapa - Cpatsa foi realizada a caracterização físico-química do solo utilizado na pesquisa (Tabela 1 e 2) conforme (EMBRAPA, 1997). Assumiu-se os valores médios de umidade na capacidade de campo pelo método da centrífuga nos potenciais mátricos de 0,006 e $-0,01 \mathrm{MPa}$. 
Tabela 1. Caracterização física do solo utilizado no experimento.

\begin{tabular}{|c|c|c|c|c|c|}
\hline \multicolumn{2}{|c|}{ Densidade } & \multirow{2}{*}{$\frac{\text { Porosidade }}{\text { Total }}$} & \multicolumn{3}{|c|}{ Granulometria } \\
\hline Solo & Partículas & & Areia & Silte & Argila \\
\hline \multicolumn{2}{|c|}{-----------kg.dm-3'------------ } & $\%$ & \multicolumn{3}{|c|}{----o- } \\
\hline 1,21 & 2,54 & 52,39 & 341,4 & 411,5 & 247,2 \\
\hline
\end{tabular}

Tabela 2. Caracterização química do solo utilizado no experimento.

\begin{tabular}{|c|c|c|c|c|c|c|c|c|c|}
\hline $\mathrm{CE}$ & $\mathrm{pH}$ & $P$ & $\mathrm{Na}$ & $\mathrm{K}$ & $\mathrm{Ca}$ & $\mathrm{Mg}$ & $\mathrm{Al}+\mathrm{H}$ & $\mathrm{V}$ & $\mathrm{MO}$ \\
\hline mS.cm-1 & & ig.dm & & - & $-\mathrm{cmc}$ & & -- & $\%$ & $\underset{1}{\mathrm{~g} . \mathrm{kg}}$ \\
\hline 0,47 & $\begin{array}{l}7,4 \\
0\end{array}$ & 1,62 & 0,1 & 0,9 & 10 & 5 & 0,2 & 99 & 8,2 \\
\hline
\end{tabular}

No ambiente protegido no Departamento de Tecnologia e Ciências Sociais (DTCS), da Universidade do Estado da Bahia (UNEB), município de Juazeiro - BA (9 ${ }^{\circ} 24^{\prime} \mathrm{S} ; 40^{\circ} 30^{\prime} \mathrm{W} ; 368 \mathrm{~m}$ de altitude) foi determinada a umidade no ponto de murcha permanente fisiológico da flor Celósia.

As plantas foram semeadas em sacos plásticos com capacidade de 1 litro previamente cheios com solo. Diariamente foram realizadas irrigações com a finalidade de manter a umidade do solo próxima a capacidade de vaso até a fase de floração quando aplicou-se o estresse hídrico, por suspensão de irrigação, cobriu-se a superfície do solo com papel alumínio, para evitar a evaporação e, quando as plantas apresentaram sinais de perda de turgescência foram encaminhadas ao final da tarde para um ambiente escuro e com umidade do ar de aproximadamente $100 \%$. No dia seguinte, ao recuperar a turgidez, as mudas foram levadas ao ambiente protegido. Este procedimento foi repetido até a murcha irreversível das plantas. Neste momento, foram coletadas amostras de solo, sem raízes, para a determinação da umidade do solo no ponto de murcha permanente fisiológico (PMPF), pelo método gravimétrico, para cada cultura sob os dois diferentes tipos de solo.

O experimento foi conduzido em delineamento inteiramente casualizado, em esquema fatorial, $2 \times 2$, com seis repetições. Sendo que os valores de umidade no ponto de murcha permanente obtidos pelos dois métodos (fisiológico e centrífuga) foram utilizados na determinação da água disponível considerando-se dois potenciais mátricos como limites superior de retenção de água $(-0,006$ e- 0,01 $\mathrm{MPa}$ ) obtidos pelo método da centrífuga. Os dados de água disponível foram submetidos à análise de variância e, quando pertinentes, as médias foram comparadas pelo teste de Tukey.

\section{RESULTADOS E DISCUSSÃO}

Na tabela 3 está apresentado o resumo da análise de variância para água disponível em função do método de determinação do ponto de murcha permanente e dos potenciais mátricos utilizados para obter a umidade na capacidade de campo.

Foi constatado, pela análise de variância a $1 \%$ de probabilidade, que o método de determinação do PMP e o potencial mátrico utilizado para determinar a CC interferiram significativamente na água disponível do solo, não se verificando o mesmo para interação método x potencial mátrico.

Observou-se maior água disponível no solo com determinação do ponto de murcha permanente pelo método fisiológico, que pelo método da centrífuga (Tabela 4). Possivelmente, devido à maior habilidade das plantas de Célosia em extraírem a água sob potencial mátrico inferior a $-1,5 \mathrm{MPa}$ (Tabela 4 e 5) ou que o equilíbrio potencial mátrico versus teor de água do solo não foi atingido nas amostras submetidas a tensão, na centrifuga, como semelhantemente foi reportado por Klein et al., (2006) em estudo sobre a quantificação da água disponível em um Latossolo Vermelho argiloso e murcha fisiológica de culturas, em que verificaram a discrepância de valores entre o ponto de murcha permanente obtido pelo método fisiológico, com girassol e milho como plantas indicadoras da murcha, e através da câmara de Richards (potencial de -1,5 MPa). Estes autores observaram que as plantas, de ambas as culturas, foram capazes de extrair água a potencial mátrico inferior a $-1,5 \mathrm{MPa}$ os quais foram encontrados por Briggs \& Shantz (1912) com girassol como cultura indicadora. 
Costa (1997) constataram que o girassol, o feijão e a soja atingiram a murcha irreversível a uma umidade maior que os determinados pela câmara de Richards no potencial mátrico de $-1,5 \mathrm{MPa}$, evidenciando que a disponibilidade de água em solos arenosos neste potencial, é menor para as culturas, determinando que elas experimentam a murcha irreversível, em potenciais mátricos maiores.

Costa et al., (2009) estudando a água disponível em um Neossolo Quartzarênico após adição de um condicionador mineral do solo verificaram diferenças estatísticas na quantificação de água disponível, em função da adição do condicionador de solo proveniente da Chapada do Araripe, contudo, observou que os valores de umidade no ponto de murcha permanente determinados pelos métodos de laboratório foram maiores que os obtidos pelo método fisiológico sem adição da dose de condicionador, indicando sensibilidade da cultura do melão ao estresse hídrico.

Tormena et al.,(1998) afirmam que sob potencial de -0,01 e -1,5 MPa a densidade exerce efeito favorável sobre a retenção de água no solo. O mesmo ainda menciona em sua obra outros autores (Gupta \& Larson, 1979; Smedema, 1993; Silva \& Kay, 1997) favoráveis a sua publicação e outros que discordam, justificando que, sob elevados potenciais, a retenção passa por severa influência da porosidade total que é inversamente proporcional a densidade, enquanto, sob baixos potenciais, a retenção é controlada pelo volume de micro-poros, os quais têm correlação positiva com a densidade do solo. Esta afirmação corrobora com a observação de Beutler et al., (2002), que em experimento com dois tipos de solo, Latossolo Vermelho distrófico e Latossolo Vermelho eutroférrico observaram que a retenção de água ocorreu de forma diferenciada nos solos, nos sistemas de uso e manejo e nas profundidades, indicando que os atributos dos solos influenciaram o conteúdo de água retido nas diferentes tensões.

Na presente pesquisa, a diferença entre os valores de ponto de murcha permanente pelo método centrifuga e fisiológico foi muito maior que o encontrado por Richards \& Weaver (1943) ao utilizar diversas culturas e mais de 100 tipos de solo e por Klein et al., (2006) com girassol como planta indicadora da murcha.

Resultados diferentes foram alcançados por Costa (1997) que não verificou diferenças estatísticas significativas entre a água disponível obtida considerando-se o ponto de murcha permanente obtido pela câmara de Richards e fisiológico para as culturas do girassol, feijão, milho, soja, dentre outras.

A água disponível diminuiu com o aumento da tensão ou do potencial mátrico utilizado para estimar a umidade na capacidade de campo independente do método de determinação do PMP, este resultado corrobora com realizao por Klein et al., (2006).

Ainda, Beutler et al.,(2002), evidenciam que mesmo que a capacidade de água disponível tenha sido a mesma nos dois solos estudados, a maior retenção sob todas as tensões ocorreu no Latossolo vermelho eutroférrico cujo textura é mais fina, que no Latossolo vermelho distrófico, demonstrando que o maior conteúdo de água neste solo não resultou em benefício para as plantas, devido ao fato de a água estar retida sob maior tensão, permanecendo indisponível para as plantas.

\section{CONCLUSÕES}

A água disponível no solo foi maior ao considerar a umidade na capacidade de campo determinada no maior potencial mátrico.

A Celósia apresentou habilidade de extração de água a potencias inferiores a $-1,5 \mathrm{MPa}$, isto incrementou a água disponível no solo.

$\mathrm{O}$ ponto de murcha permanente foi significativamente menor no método fisiológico que o determinado na Centrífuga.

\section{REFERENCIAS BIBLIOGRAFICAS}

BEUTLER, A. N.; CENTURION, J. F.; SOUZA, Z. M.; ANDRIOLI, I.; ROQUE, C. G. Retenção de água em dois tipos de Latossolos sob diferentes usos. Revista Brasileira de Ciências do Solo, 26:829-834, 2002

BRIGGS, L. J.; SHANTZ, H. L. The wilting coefficient for different plants and its indirect determination. Washington: Department of Agriculture. 1912. 83p. Bulletin 230 
CARLESSO, REIMAR. Absorção de água pelas plantas: água disponível versus extraível e a produtividade das culturas. Ciência Rural, Santa Maria, v. 25, n. 1, 1995.

CASSEL, D. K.; NIELSEN, D. R. Field capacity and available water capacity. In: Klute, A. (ed.) Methods of soil analysis. Part 1 -Physical and mineralogical methods. 2.ed. Madison: SSSA, 1986, p.901-926. Book Series, 5.

COLMAN, E. A. A laboratory procedure for determining the field capacity of soils. Soil Science, v.63, p.277-283, 1947. http://dx.doi.org/10.1097/00010694-194704000-00003

COSTA, C. S.; RODRIGUES, J. J. V; CAVALCANTI, A. C.; MARINHO, L. B.; ALMEIDA NETO, L. A.; SILVA, T. J. A.. Água disponível em um Neossolo Quartzarênico após adição de um condicionador mineral do solo. Revista Brasileira de Engenharia Agrícola e Ambiental, Campina Grande, v.13, n.5, p.524-530, 2009.

COSTA, A. C. S. da; NANNI, M. R.; JESKE, E. Determinação da umidade na capacidade de campo e ponto de murchamento permanente por diferentes metodologias. Revista UNIMAR, v.1, n.3, p.827844, 1997.

EMBRAPA - Empresa Brasileira de Pesquisa Agropecuária. Centro Nacional de Pesquisa de Solos. Manual de métodos de análise de solo. 2.ed. Rio de Janeiro: Embrapa CNPS, 1997. 212p

GUPTA, S.C.; LARSON, W.E. Estimating soil water characteristics from size distribution, organic carbon and bulk density. Water Res. Res., 15:1633-1635, 1979 http://dx.doi.org/10.1029/WR015i006p01633

KLEIN, V. A.; REICHERT, J.M.; REINERT, D. J. Água disponível em um Latossolo Vermelho argiloso e murcha fisiológica de culturas. Revista Brasileira de Engenharia Agrícola e Ambiental, Campina Grande, v. 10, n.3,2006.

RICHARDS, L. A.; WEAVER, L. R. Fitten-atmosphere percentage as related to the permanent wilting percentage. Soil Science, v.56, p.331-339, 1943. http://dx.doi.org/10.1097/00010694-19431100000002

RIVERS, E. D.; SHIPP, R. F. Soil water retention as related to particle size in selected sands and loamy sands. Soil Science, v.126, p.94-100, 1978. http://dx.doi.org/10.1097/00010694-19780800000005

SALVESTRO, A. C.; FREITAS, P. S. L. de; REZENDE, R.; VIEIRA, C. V.;.FRISKE, E.; CHIERICI. G.; SAPIA, J. G.; BARBOSA, A. PRECHLAK.Permanent wilting point of soybean in Rhodic Ferralsols with and without biodegradable organic residue incorporation and physiological monitoring in relation to the behavior of moisture. Journal of Food, Agriculture \& Environmen, v.11, n.3-4, p. 1248-1252, 2013.

SILVA, A.P.; KAY, B.D. Estimating the least limiting water range of soil from properties and management. Soil Sci. Soc. Am. J., 61:877-883, 1997. http://dx.doi.org/10.2136/sssaj1997.03615995006100030023x

SMEDEMA, L.K. Drainage performance and soil management. Soil Technol., 6:183-189, 1993. http://dx.doi.org/10.1016/0933-3630(93)90007-2

TORMENA, C. A.; SILVA, A. P.; LIBARDI, P. L. Caracterização do intervalo hídrico ótimo de um latossolo roxo sob plantio direto. Revista Brasileira de ciências do Solo, 22:573-581, 1998

Tabela 3. Análise variância para água disponível no solo.

\begin{tabular}{llll}
\hline Causa variação & G. L & Q. M & F \\
\hline Método & 1 & 214,68 & $1152,87^{* *}$ \\
Potencial mátrico & 1 & 49,91 & $268,04^{* *}$ \\
Interação & 1 & 0,0041 & $0,02^{\text {ns }}$ \\
\hline
\end{tabular}

** significativo a $1 \%$ de probabilidade 
Tabela 4. Valores de ponto de murcha permanente fisiológico e água disponível com a capacidade de campo obtidas nos potenciais mátricos de $-0,006$ e $-0,01 \mathrm{MPa}$.

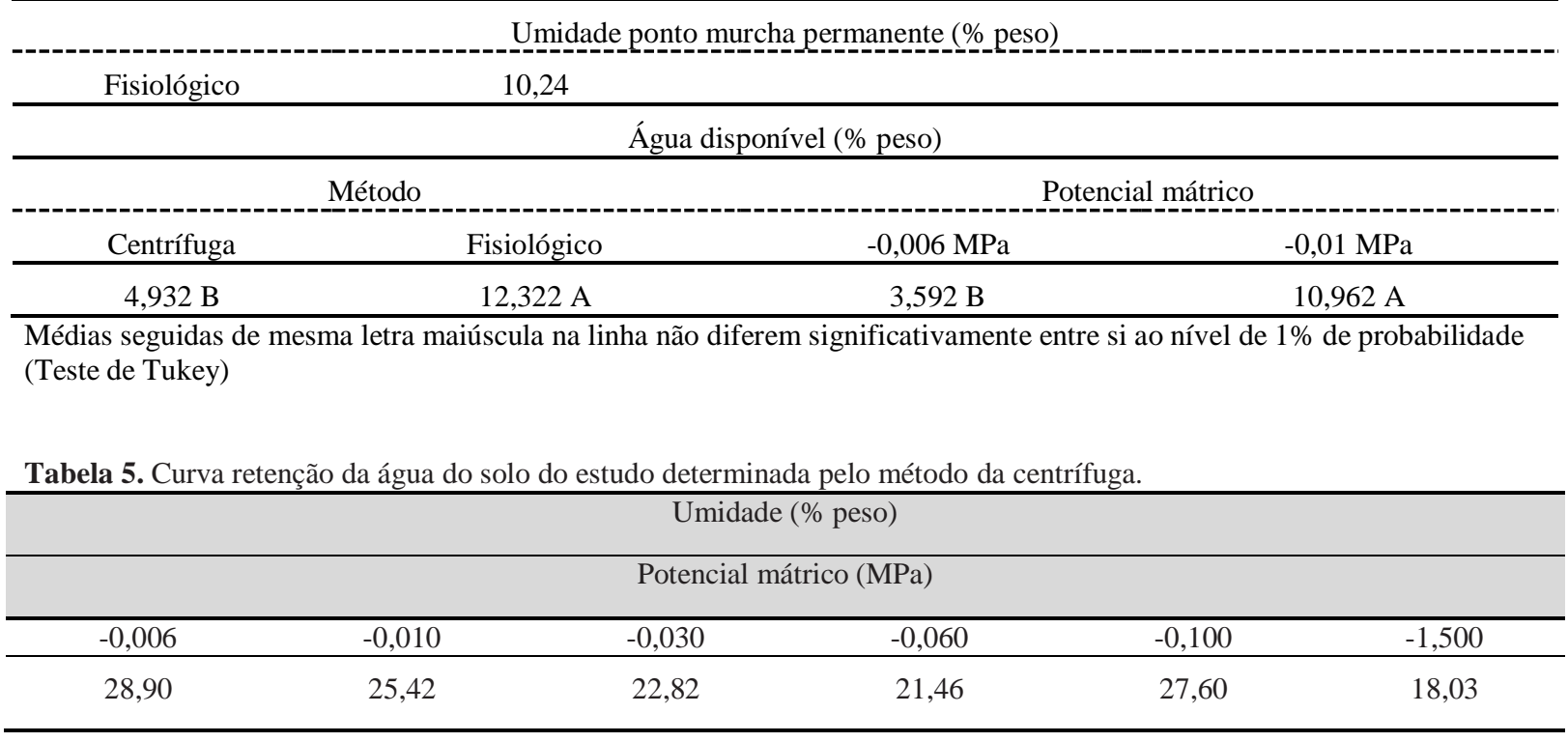

